\title{
THE WEAK SURVIVAL/STRONG SURVIVAL PHASE TRANSITION FOR THE CONTACT PROCESS ON A HOMOGENEOUS TREE
}

\author{
STEVEN P. LALLEY AND THOMAS M. SELLKE
}

\section{INTRODUCTION}

The contact process on a homogeneous tree $\mathcal{T}_{d}$ of degree $d+1 \geq 3$ is known $[10,7,13]$ to have three distinct phases: an extinction phase, a weak survival phase, and a strong survival phase. The existence of two qualitatively different survival phases is the most striking feature of the process, as the contact process on the integer lattice $\mathbb{Z}^{d}$, in any dimension, exhibits only one survival phase (strong survival). Thus, the contact process on a homogeneous tree exhibits a phase transition, from weak to strong survival, of a different character than the phase transition for the contact process on the integer lattices. The purpose of this paper is to speculate on the nature of this phase transition, and to show how certain conjectured behavior of the expected total infection time in the weak survival phase would delimit the critical exponent of the "Malthusian parameter" $\beta d$ defined by (1) below.

In the weak survival phase, the contact process, when started from a single infected site (by convention, the root vertex $r$ of the tree), survives forever with positive probability, but with probability one eventually vacates every finite subset of the tree. For any vertex $x$ other than the root, the probability of eventual infection is less than one. This probability $u_{x}=u_{n}$ depends only on the distance $n=|x|$ from $r$ to $x$, and decays exponentially in $n$; the decay rate is

$$
\beta:=\lim _{n \rightarrow \infty} u_{n}^{1 / n} .
$$

This rate is of interest in part because it determines the Hausdorff dimension (relative to the natural metric on the space of ends of the tree - see [5] for details) of the limit set $\Lambda$ (the set of ends of the tree in which the infection survives):

$$
H D(\Lambda)=\frac{\log (\beta d)}{\log 2}
$$

almost surely on the event of survival. Equivalently, the subtree consisting of vertices ever infected has branching number $\log (\beta d)$ (see [9] for the definition); hence, $\beta d$ serves as a Malthusian parameter for the contact process.

Date: April 8, 2002. 
It is known [5] that, in the weak survival phase,

$$
\beta \leq 1 / \sqrt{d},
$$

and so the Hausdorff dimension of the limit set $\Lambda$ can never be more than half the Hausdorff dimension of the space of ends. Since $\beta$ is left-continuous in the infection rate parameter, it follows that the contact process survives only weakly at the weak/strong survival transition, and that $\beta$ is discontinuous at the critical point. It is not yet known if $\beta=1 / \sqrt{d}$ at any values of the infection and recovery rate parameters other than at the critical point, but it is known [4] that if $\beta<1 / \sqrt{d}$ then an increase in the infection rate (or a decrease in the recovery rate) will strictly increase the value of $\beta$.

Denote by $\lambda$ and $\delta$ the infection and recovery rates of the contact process. Recall that, for any $\epsilon>0$, the contact process with infection and recovery rates $\epsilon \lambda$ and $\epsilon \delta$ is a time-changed version of the contact process with rates $\lambda$ and $\delta$, and observe that this time change has no effect on the limit set $\Lambda$ or the parameter $\beta$. It is customary to set $\delta=1$, and to let $\lambda$ vary; however, we shall find it more convenent to fix $\lambda=1$, and to let $\delta$ vary. The critical points will be denoted by $\delta_{u}$ and $\delta_{c}$ : thus, $\delta<\delta_{u}$ is the strong survival phase; $\delta_{u} \leq \delta<\delta_{c}$ is the weak survival phase; and $\delta \geq \delta_{c}$ is the extinction phase. Our main conjecture is that the critical exponent for the parameter $\beta$ at the weak/strong survival transition is $1 / 2$ :

\section{Conjecture 1.}

$$
\lim _{\delta \downarrow \delta_{u}} \frac{\log (1 / \sqrt{d}-\beta(\delta))}{\log \left(\delta-\delta_{u}\right)}=\frac{1}{2}
$$

Notational Conventions: The set of infected sites at time $t$ in a contact process started at time 0 with only the root vertex initially infected will be denoted by $\xi_{t}$ or $\zeta_{t}$. The values of constants will not be carefully delineated: thus, $C$ may denote different constants from one inequality to the next.

\section{Expected Total Infection Time}

Weak survival differs from strong survival in that, with probability one, every vertex $x$ is eventually healthy, and so the total infection time at vertex $x$ is finite. It does not necessarily follow that the expected total infection time is finite; however, this must be the case if $\beta<1 / \sqrt{d}$, because it is known [3] that if $\beta<1 / \sqrt{d}$ then $P\left\{r \in \xi_{t}\right\}$ decays exponentially in $t$. Because the contact process survives only weakly at the critical point, and because the hitting probability $u_{n}$ decays exponentially in $n$ even at the critical point, it is natural to expect that the conditional expectation of the total infection time for any vertex, given that it is positive, remains bounded. Denote by $J(x)$ the total infection time at $x$, that is,

$$
J(x)=\int_{0}^{\infty} \mathbf{1}\left\{x \in \xi_{t}\right\} d t .
$$


Conjecture 2. There exists a constant $C=C_{d}$ depending only on the degree $d+1$ of the tree $\mathcal{T}_{d}$ such that, for every vertex $x$ and all values $\delta$ of the recovery parameter such that $\delta \geq \delta_{u}$,

$$
E(J(x) \mid J(x)>0) \leq C .
$$

This conjecture is largely motivated by the fact that the analogous statement is true for the isotropic, nearest-neighbor branching random walk on $\mathcal{T}_{d}$, whose behavior in the weak survival phase resembles in many other respects [8] that of the contact process. In this case, that $C<\infty$ follows from the fact that $G(R)<\infty$, where $G(z)$ is the Green's function of the underlying random walk and $R$ is its radius of convergence; that $G(R)<\infty$ is a consequence of the nonamenability of $\mathcal{T}_{d}$, which precludes the possibility of $R$-recurrence for any nondegenerate random walk on $\mathcal{T}_{d}$.

The expected total infection time at a vertex $x$ is comparable to several related quantities. Recall that the contact process may be constructed from a percolation structure, a system of independent Poisson processes attached to vertices and ordered pairs of neighboring vertices. The Poisson processes attached to vertices have intensity $\delta$; their occurrences mark the times of recoveries from infection. The Poisson processes attached to ordered pairs $(x, y)$ of neighboring vertices are of rate 1 ; their occurrences, which we shall call infection arrows, or simply arrows, mark the times at which infection may pass from $x$ to $y$. The set $\xi_{t}$ of infected sites at time $t$ in the contact process started in state $\xi_{0}=\{r\}$ consists of those vertices $y$ such that there is a path (called an infection trail) in the percolation structure starting at $r$ at time 0 and terminating at $y$ at time $t$ (this path may cross arrows in the percolation structure, in the direction of the arrows, but may not pass through recovery marks). Define $M^{+}(x)$ (respectively, $M^{-}(x)$ ) to be the number of infection arrows $\alpha$ with head (respectively, tail) $x$ such that there is an infection trail starting at $r$ at time 0 that passes through $\alpha$. Similarly, define $N(x)$ to be the number of recovery marks at $x$ that mark the end of time intervals in which $x \in \xi_{t}$.

Lemma 3. There exist constants $C_{1}, C_{2}, C_{3}, C_{4}<\infty$, independent of the recovery rate $\delta$, such that for every vertex $x$ and all values of $\delta \geq \delta_{u}$ near $\delta_{u}$

$$
\begin{aligned}
E(J(x) \mid J(x)>0) & \leq C_{1} E(N(x) \mid J(x)>0) \\
& \leq C_{2} E\left(M^{+}(x) \mid J(x)>0\right) \\
& \leq C_{3} E\left(M^{-}(x) \mid J(x)>0\right) \\
& \leq C_{4} E(J(x) \mid J(x)>0) .
\end{aligned}
$$

Proof. These inequalities follow by arguments very similar to those used in $[12]$. 
Corollary 4. If Conjecture 2 is true, then there are constants $0<C_{1}<$ $1<C_{2}<\infty$ such that for every vertex $x$ and all values of $\delta \geq \delta_{u}$ near $\delta_{u}$,

$$
\begin{gathered}
C_{1} \beta^{|x|} \leq u_{x} \leq \beta^{|x|} \quad \text { and } \\
\beta^{|x|} \leq E N(x) \leq C_{2} \beta^{|x|} .
\end{gathered}
$$

Proof. Since the function $u_{x}=u_{|x|}$ is supermultiplicative in $|x|$, it follows from Fekete's subadditivity lemma and (1) that $u_{x} \leq \beta^{|x|}$ for all $x$. Similarly, it is easily seen that $E^{+}(x)$ is submultiplicative in $|x|$;, according to Theorem 2 of [12], the exponential decay rate of $E M^{+}(x)$ in $|x|$ is also $\beta$, and so $E M^{+}(x) \geq \beta^{|x|}$. Now $E M^{+}(x) \geq u_{x}$, because in order that $x$ be infected at some time there must be at least one infection arrow leading to $x$. Finally, by Lemma 3, Conjecture 2 implies that, for a suitable finite constant $C$,

$$
E\left(M^{+}(x) \mathbf{1}\{J(x)>0\}\right) \leq C E \mathbf{1}\{J(x)>0\}=C u_{x} .
$$

The inequality $E N(x) \leq C^{\prime} u_{x}$, for a suitable constant $C^{\prime}<\infty$, now follows from Lemma 3.

\section{Critical Exponent for the Malthusian Parameter}

As noted earlier, it is as yet unknown whether $\beta(\delta)<1 / \sqrt{d}$ for all $\delta \geq \delta_{u}$, although this is believed to be the case, for the following reason: As proved in [3] strict inequality $\beta<1 / \sqrt{d}$ in (1) holds if and only if $P\left\{r \in \xi_{t}\right\}$ decays exponentially in $t$. Thus, if it were the case that $\beta=1 / \sqrt{d}$ for some $\delta>\delta_{u}$, then it would follow that $P\left\{r \in \xi_{t}\right\}$ decays subexponentially in $t$ and that the contact process stays in the weak survival regime when $\delta$ is relaxed. This seems unlikely. In any case, we may define

$$
\delta_{*}=\max \left\{\delta \geq \delta_{u}: \beta(\delta)=1 / \sqrt{d}\right\} .
$$

Theorem 1. If Conjecture 2 is true, then there is a finite constant $C=C_{d}$ such that for all $\delta>\delta_{*}$ near $\delta_{*}$,

$$
1 / \sqrt{d}-\beta(\delta) \leq C \sqrt{\delta-\delta_{*}} .
$$

Thus, if Conjecture 2 is true, and if there is a critical exponent for the decay rate $\beta$ at the critical point $\delta_{*}$, then it cannot be less than $1 / 2$. The proof outlined below also suggests that $1 / 2$ is the correct value, as the inequalities in the proof are very likely approximate equalities.

The proof of Theorem 1 will make use of the following lemma, proved in $[12]$.

Lemma 5. The decay rate $\beta$ varies continuously with the recovery rate parameter $\delta$ for $\delta \geq \delta_{u}$. 
Proof of Theorem 1. We shall estimate the change in $\beta$ that results when the recovery rate $\delta$ is decreased to $(1-\epsilon) \delta$ for small $\epsilon$. For this, we shall construct versions $\xi_{t}$ and $\zeta_{t}$ of the contact processes with initial states $\xi_{0}=\zeta_{0}=\{r\}$ and recovery rates $\delta$ and $(1-\epsilon) \delta$, respectively, using a common augmented percolation structure. The base percolation structure, used for constructing $\xi_{t}$, is as described in Section 2: the intensities of the arrow processes and the recovery mark processes are 1 and $\delta$, respectively, and these processes are mutually independent Poisson processes. This base percolation structure is augmented by attaching to each recovery mark (at every vertex) a Bernoulli- $\epsilon$ random variable; these random variables are mutually independent, and independent of the arrow and recovery mark processes. Those recovery marks for which the attached Bernoulli takes the value 1 are colored GREEN, and those not colored GREEN are colored RED. The base percolation structure is now modified by removing all the GREEN recovery marks, and a version $\zeta_{t}$ of the contact process with recovery rate $(1-\epsilon) \delta$ is obtained by proceeding in the usual manner, as described in Section 2, but using the modified percolation structure. Since the set of recovery marks obtained by removing the GREEN marks is contained in the set of recovery marks in the base percolation structure, every infection trail in the base percolation structure remains an infection trail in the modified percolation structure; therefore,

$$
\xi_{t} \subseteq \zeta_{t} \quad \forall t \geq 0 .
$$

Let $x_{0}=r, x_{1}, x_{2}, \ldots$ be the vertices along a fixed (but arbitrary) geodesic ray emanating from the root of the tree, so that $\left|x_{n}\right|=n$ for each $n \geq 0$. Denote by $u_{n}(\delta)$ and $u_{n}(\delta-\delta \epsilon)$ the hitting probabilities of vertex $x_{n}$ for the processes $\xi_{t}$ and $\zeta_{t}$, respectively. In view of (11), it must be the case that $u_{n}(\delta) \leq u_{n}(\delta-\delta \epsilon)$, and the discrepancy must be

$$
u_{n}(\delta-\delta \epsilon)-u_{n}(\delta)=P\left\{x_{n} \in \cup_{t \geq 0} \zeta_{t} \backslash \cup_{t \geq 0} \xi_{t}\right\}:=P\left(F_{n}\right) .
$$

Now in order that event $F_{n}$ occur, it is necessary that in the modified percolation structure (that is, the percolation structure obtained by removing the GREEN recovery marks) there should be an infection trail $\mathcal{I}$ from the root, starting at $t=0$, that ends at $x_{n}$, but that in the base percolation structure there should be no such infection trail. On this event, the infection trail $\mathcal{I}$ must pass through at least one GREEN recovery mark, because otherwise it would be an infection trail in the base percolation structure. Thus, the discrepancy (12) is no larger than the probability that there is an infection trail $\mathcal{I}$ from $(r, 0)$ to $x_{n}$ in the modified percolation structure that passes through at least one GREEN recovery mark, and so

$$
u_{n}(\delta-\delta \epsilon)-u_{n}(\delta) \leq E K_{n},
$$

where $K_{n}$ is defined to be the number of GREEN recovery marks in the augmented percolation structure that lie on infection trails from $(r, 0)$ to $x_{n}$ on which there are no earlier GREEN marks. 
Lemma 6. If Conjecture 2 is true, then there exists a constant $C<\infty$ such that for all values of $\delta>\delta_{u}$ and $\epsilon>0$ such that $\delta-\delta \epsilon>\delta_{u}$, and all $n=1,2, \ldots$,

$$
E K_{n} \leq C \epsilon n \beta^{n} /\left(1-d \beta^{2}\right),
$$

where $\beta=\beta(\delta-\delta \epsilon)$.

Observe that $1-d \beta^{2}>0$ for all $\delta>\delta_{*}$, by (3). Note also the affinity of the inequalities (13)-(14), which relate the derivative $d u_{n} / d \delta$ to an expected count, with Russo's formula ([1], Section 2.4 and [11]) inercolation theory. Here, however, the objects being counted cannot be interpreted as "pivotal" in the sense of [1] and [11].

Before proving Lemma 6 we will show how it implies Theorem 1. First, we show that the inequality (14) forces an upper bound on the derivative of $\beta$ with respect to $\delta$ :

Corollary 7. The derivative $d \beta / d \delta$ exists at almost every $\delta>\delta_{*}$. Furthermore, if Conjecture 2 is true, then there is a constant $C<\infty$ such that for almost all $\delta>\delta_{*}$ near $\delta_{*}$,

$$
\frac{d \beta}{d \delta} \leq \frac{C}{1-d \beta^{2}}
$$

Note: The $d$ attached to $\beta^{2}$ on the right side of (15) is the degree of the tree minus 1 , whereas the $d$ 's on the left side indicate derivatives with respect to $\delta$.

Proof. Since $u_{n}$ and $\beta$ are monotone and continuous in $\delta>\delta_{*}$, they are differentiable at almost every value of $\delta$. The inequalities (13) and (14) imply that the derivative of $u_{n}(\delta)$ with respect to to $\delta$, where it exists, must satisfy

$$
\frac{d u_{n}}{d \delta} \leq C^{\prime} n \beta^{n} /\left(1-d \beta^{2}\right)
$$

where $C^{\prime}=C / \delta_{*}$ and $C$ is as in Lemma 6 . If Conjecture 2 holds, then by Corollary 4 there is a positive constant $c$ such that $u_{n} \geq c \beta^{n}$ for all values $\delta \geq \delta_{*}$ near $\delta_{*}$ and all $n \geq 1$; hence, dividing both sides of inequality (16) by $n u_{n}$ yields

$$
\frac{d \log u_{n}^{1 / n}}{d \delta} \leq \frac{C^{\prime \prime}}{1-d \beta^{2}}
$$

where $C^{\prime \prime}=C^{\prime} / c$. Integrating this over the interval $\left[\delta_{1}, \delta_{2}\right]$ and letting $n \rightarrow \infty$, using (1), we obtain

$$
\log \frac{\beta\left(\delta_{1}\right)}{\beta\left(\delta_{2}\right)} \leq C^{\prime \prime} \int_{\delta_{1}}^{\delta_{2}} \frac{d \delta}{1-d \beta^{2}} .
$$

Since $\beta$ is continuous in $\delta$ for $\delta \geq \delta_{*}$, and since $d \beta^{2}<1$ for all $\delta>\delta_{*}$, inequality (18) implies that the derivative of $\log \beta$, where it exists, is bounded 
above by $C^{\prime \prime} /\left(1-d \beta^{2}\right)$. By the chain rule, it follows that the derivative of $\beta$, where it exists, is bounded above by $C /\left(1-d \beta^{2}\right)$, for a suitable constant $C$.

Proof of Theorem 1. Let $\gamma=d \beta^{2}$ denote the Malthusian parameter. By Corollary 7 , if Conjecture 2 is true then for a suitable constant $C^{\prime}<\infty$,

$$
\frac{d \gamma}{d \delta} \leq \frac{C^{\prime}}{1-\gamma}
$$

for almost every $\delta>\delta_{*}$ near $\delta_{*}$. This inequality may be integrated between $\delta_{*}$ and $\delta$, using the fact that $\gamma \rightarrow 1$ as $\delta \rightarrow \delta_{*}$ (by definition of $\delta_{*}$ ). The result is that, for all $\delta>\delta_{*}$ near $\delta_{*}$,

$$
(1-\gamma)^{2} \leq C^{\prime \prime}\left(\delta-\delta_{*}\right)
$$

where $C^{\prime \prime}=C^{\prime} / 2$. Inequality (10) now follows by taking square roots.

Proof of Lemma 6. Recall that $K_{n}$ is defined to be the number of GREEN recovery marks in the augmented percolation structure that lie on infection trails from $(r, 0)$ to $x_{n}$ on which there are no earlier GREen marks. This may be decomposed as a disjoint sum, by grouping GreEn recovery marks according to their locations in the tree: For each integer $m \geq 0$, define $H_{m}$ to be the set of all vertices $x$ such that the geodesic path from $r$ to $x$ passes through $x_{m}$ but not $x_{m+1}$ (recall that $x_{0}, x_{1}, x_{2}, \ldots$ are the vertices along a fixed but arbitrary geodesic ray emanating from the root), and set $G_{m}=\cup_{k \leq m} H_{k}$. Then

$$
K_{n}=\sum_{x \in G_{n}} K_{n}^{x}
$$

where $K_{n}^{x}$ is the number of GREEN recovery marks at $x$ accessible by infection trails in the base percolation structure starting at $(r, 0)$ and from which emanate infection trails in the modified percolation structure terminating at $x_{n}$.

Recall that $N(x)$ is the number of recovery marks in the base percolation structure at $x$ where infection trails starting at $(r, 0)$ terminate. For each such recovery mark, there is probability $\epsilon$ that the mark will be colored Green in the Bernoulli thinning. Moreover, for each such mark, the conditional probability that it initiates an infection trail in the modified percolation structure terminating at $x_{n}$, given the history of the percolation structure up to the time of the mark, is $u_{k}(\delta-\delta \epsilon)$, where $k$ is the distance from $x$ to $x_{n}$, and so is bounded above by $\beta^{k}$ (where $\beta=\beta(\delta-\delta \epsilon)$ ). Hence,

$$
E K_{n}^{x}=u_{k}(\delta-\delta \epsilon) \epsilon E N(x) \leq \beta^{k} \epsilon E N(x) .
$$

If Conjecture 2 holds then, by Corollary 4 , there is a constant $C<\infty$ such that $E N(x) \leq C \beta^{|x|}$ for every vertex $x$ and all values $\delta>\delta_{u}$. Consequently, by (21), $E K_{n}^{x}$ is bounded above by $C \epsilon \beta^{k+l}$, where $l=|x|$ and $k$ is the distance from $x$ to $x_{n}$. Now if $x \in H_{m}$ for some $0 \leq m \leq n$, and if the 
distance from $x$ to $x_{m}$ is $j$, then $l+k=n+2 j$. Since the number of vertices $x \in H_{m}$ at distance $j$ from $x_{m}$ is at most $d^{j}$, it follows that

$$
\begin{aligned}
E K_{n} & =\sum_{x \in G_{n}} E K_{n}^{x} \\
& =\sum_{m=0}^{n} \sum_{x \in H_{m}} E K_{n}^{x} \\
& \leq \sum_{m=0} \sum_{j=0}^{\infty} d^{j} C \epsilon \beta^{n+2 j} \\
& =\sum_{m=0}^{n} C \epsilon \beta^{n} /\left(1-d \beta^{2}\right) \\
& \leq(n+1) C \epsilon \beta^{n} /\left(1-d \beta^{2}\right) .
\end{aligned}
$$

\section{REFERENCES}

[1] Grimmett, G. (1989) Percolation. Springer-Verlag, New York.

[2] Harris, T. E. (1978) Additive set-valued Markov processes and percolation methods. Annals of Probability 6 355-378.

[3] Lalley, S. (1999) Growth profile and invariant measures for the weakly supercritical contact process on a homogeneous tree. Annals of Probability 27 206-225

[4] Lalley, S. (1999) Correction to "Growth profile and invariant measures for the weakly supercritical contact process on a homogeneous tree." Preprint available at http://galton.uchicago.edu/ lalley/Papers.

[5] Lalley, S. and Sellke, T. (1998) Limit set of a weakly supercritical contact process on a homogeneous tree. Annals of Probability 26 644-657

[6] Liggett, T. (1985) Interacting Particle Systems. Springer-Verlag, New York

[7] Liggett, T. (1996) Multiple transition points for the contact process on the binary tree. Annals of Probability 24 1675-1710.

[8] Liggett, T. (1996) Branching random walks and contact processes on homogeneous trees. Probability and Related Fields 106 495-519.

[9] Lyons, R. (1990) Random walks and percolation on trees. Annals of Probability 18 931-958.

[10] Pemantle, R. (1992) The contact process on trees. Annals of Probability 202089 2116.

[11] Russo, L. (1981) On the critical percolation probabilities. Z. Warscheinlichkeitstheorie 56 229-237.

[12] Schonmann, R. (1998) The triangle condition for contact processes on homogeneous trees. J. Statistical Physics 90 1429-1440.

[13] Stacey, A. M. (1996) Existence of an intermediate phase for the contact process on trees. Annals of Probability 24 1711-1726. 
University of Chicago, Department of Statistics, 5734 University Avenue, Chicago IL 60637

E-mail address: lalley@galton.uchicago.edu

Purdue University, Department of Statistics, Mathematical Sciences BuildING, W. LAFAYETTE IN 47907

E-mail address: tsellke@odds.stat.purdue.edu 